P. Brekke, B. Fleck, and J. B. Gurman eds.

\title{
Vorticity, Current Helicity and Alpha-effect for Magnetic-driven Turbulence in the Solar Convection Zone
}

\author{
G. Rüdiger
}

\begin{abstract}
Astrophysikalisches Institut Potsdam, An der Sternwarte 16, 14482 Potsdam, Germany
\end{abstract}

\begin{abstract}
The turbulent electromotive force as well as the kinetic and current helicities have been computed for a turbulence subject to magnetic buoyancy and global rotation. The dynamo-alpha is found as positive in the northern hemisphere and negative in the southern hemisphere and the kinetic helicity has just the same signs.

In agreement with the observations the current helicity is negative in the northern hemisphere and positive in the southern hemisphere. Our current helicities and alpha-effects are thus always out of phase. The signs of alpha-effect and both helicities exactly correspond to a numerical simulation by Brandenburg \& Schmitt (1998).
\end{abstract}

\section{Key quantities}

The mean-field theory of the the magnetic solar activity is defined by a couple of mean-field quantities characterising the turbulence. Important, e.g., is the relation of the turbulent electromotive force (EMF) to a uniform mean magnetic field,

$$
\left\langle\boldsymbol{u}^{\prime} \times \boldsymbol{B}^{\prime}\right\rangle=\alpha_{\mathrm{dyn}} \circ \overline{\boldsymbol{B}}
$$

As known the $\alpha_{\text {dyn }}$ is a pseudotensor with different signs in the both hemispheres. In order to reproduce the solar butterfly diagram the relation $\alpha_{\mathrm{dyn}}^{\text {north }}<0$ must be fulfilled. Important are also both the helicities $\mathcal{H}_{\text {kin }}=\left\langle\boldsymbol{u}^{\prime} \cdot \operatorname{rot} \boldsymbol{u}^{\prime}\right\rangle$ and $\mathcal{H}_{\text {curr }}=$ $\left\langle j^{\prime} \cdot \boldsymbol{B}^{\prime}\right\rangle$ due to their close relation to the $\alpha$-effect and due to the possibility to observe them at the solar surface.

Even more complicated is the observation of the kinetic helicity as the mesogranulation only 'feels' a very weak rotational influence. Helpful is the 'vortex-divergence correlation'

$$
\mathcal{C}=\left\langle\left(\frac{\partial u}{\partial x}+\frac{\partial v}{\partial y}\right)\left(\frac{\partial v}{\partial x}-\frac{\partial u}{\partial y}\right)\right\rangle
$$

where only horizontal flow components $u$ (east) and $v$ (north) are involved. With $\mathcal{C} \simeq \mathcal{H}_{\text {kin }} / H_{\mathrm{m}}$ and the positive momentum scale height $H_{\mathrm{m}}$ the correlation $\mathcal{C}$ is a helicity proxy (Rüdiger, Pipin, \& Belvedere 2000). For mesogranulation the observations seem to indicate $\mathcal{C}^{\text {north }} \lesssim 0$ (Brandt et al. 1988; Wang et al. 1995; Duvall \& Gizon 2000) so that $\mathcal{H}_{\text {kin }}^{\text {north }} \lesssim 0$. Again the $\alpha$-effect proves to be positive at the northern hemisphere. 


\section{The canonical theory}

Everything is simple in the traditional theory where the turbulence is considered as driven by forces and as subject to a global rotation and a density stratification. There are then two simple and well-known relations, i.e.

$$
\alpha_{\phi \phi} \simeq-\tau_{\text {corr }} \mathcal{H}_{\text {kin }}
$$

and

$$
\mathcal{H}_{\text {kin }} \simeq \tau_{\text {corr }}(\boldsymbol{g} \cdot \boldsymbol{\Omega}) \lessgtr 0\left\{\begin{array}{l}
\text { north } \\
\text { south }
\end{array} .\right.
$$

The $\alpha$-effect is thus positive (negative) at the northern (southern) hemisphere in contrast to the above mentioned condition. One can also find the relation

$$
\mathcal{H}_{\text {curr }} \simeq \tau_{\text {corr }} B_{\phi}^{2} \mathcal{H}_{\text {kin }}
$$

between both the helicities. The main point here is that the current helicity can be observed with much more ease than the kinetic helicity (Hale 1927; Seehafer 1990; Pevtsov, Canfield, \& Metcalf 1995; Abramenko, Wang, \& Yurchishin 1996; Bao \& Zhang 1998). Always the result is that the current helicity is negative (positive) at the northern (southern) hemisphere, leading again to a positive (negative) $\alpha$-effect at the northern (southern) hemisphere.

\section{Buoyancy-driven turbulence}

The turbulence model is now modified. Magnetic buoyancy is included and the turbulence is driven by magnetic fluctuations (Rüdiger, Pipin, \& Belvedere 2000). The momentum conservation then is described by

$\frac{\partial \boldsymbol{u}^{\prime}}{\partial t}+\boldsymbol{\Omega} \times \boldsymbol{u}^{\prime}+s \cdot \Omega \frac{\partial \boldsymbol{u}^{\prime}}{\partial \phi}=-\frac{1}{\rho} \operatorname{grad}\left(p^{\prime}+\frac{\boldsymbol{B}^{(0)} \cdot \overline{\boldsymbol{B}}}{\mu_{0}}\right)+\frac{\rho^{\prime}}{\rho} \boldsymbol{g}+\frac{1}{\mu_{0} \rho}(\overline{\boldsymbol{B}} \cdot \nabla) \boldsymbol{B}^{(0)}+\nu \Delta \boldsymbol{u}^{\prime}$,

with $s=r \sin \theta$. We do not use the anelastic approximation. Mass conservation is via $\partial \rho^{\prime} / \partial t+\bar{\rho} \operatorname{div} u^{\prime}=0$ (note the uniform density), and the energy equation is simply $p^{\prime}=c_{\mathrm{ac}}^{2} \rho^{\prime}$, where $c_{\mathrm{ac}}$ is the speed of sound. For the induction equation the linear but nonaxisymmetric form is used, i.e.

$$
\frac{\partial \boldsymbol{B}^{\prime}}{\partial t}+s \Omega \frac{\partial \boldsymbol{B}^{\prime}}{\partial \phi}-\eta \Delta \boldsymbol{B}^{\prime}=\operatorname{rot}\left(\boldsymbol{u}^{\prime} \times \overline{\boldsymbol{B}}\right) .
$$

\section{Results}

For the current helicity the expression

$$
\mathcal{H}_{\text {curr }}=\frac{2}{5}(\boldsymbol{g} \cdot \boldsymbol{\Omega}) \mathrm{Mm}^{2} I_{1} \lessgtr 0\left\{\begin{array}{l}
\text { north } \\
\text { south }
\end{array}\right.
$$


results with the magnetic Mach number $\mathrm{Mm}=\bar{B}^{2} / \mu_{0} \rho / c_{\mathrm{ac}}^{2} \simeq 1$. The integral

$$
I_{1}=\frac{1}{\mu_{0}} \iint \frac{\eta k^{2}\left(\nu^{2} k^{4}+\omega^{2}\right)-2 \omega^{2} \nu k^{2}}{\left(\omega^{2}+\nu k^{4}\right)^{2}\left(\omega^{2}+\eta^{2} k^{4}\right)} k^{2} \mathcal{B}(k, \omega) d \boldsymbol{k} d \omega
$$

with the positive-definite spectrum $\mathcal{B}$ of the magnetic fluctuations is positive for a cell structure of the magnetic fluctuations close to the pattern modelled by the mixing-length theory. Then the signs given in (7) follow. Next we restrict ourselves to consider the main component of the $\alpha$-effect producing the poloidal field. It proves to be

$$
\alpha_{\phi \phi}=-\frac{1}{5}(\boldsymbol{g} \cdot \boldsymbol{\Omega}) \frac{I_{2}}{c_{\mathrm{ac}}^{2}}
$$

with

$$
I_{2}=\frac{1}{\mu_{0} \rho} \iint \frac{\nu^{2} k^{4}-\omega^{2}}{\left(\omega^{2}+\nu^{2} k^{4}\right)^{2}} \mathcal{B}(k, \omega) d \boldsymbol{k} d \omega,
$$

which again is positive for reasonable spectral function $\mathcal{B}$. The $\alpha$-effect is thus positive (negative) at the northern (southern) hemisphere. Finally, for the kinetic helicity we find

$$
\mathcal{H}_{\text {kin }} \simeq-\mathrm{Mm}^{2}(\boldsymbol{g} \cdot \boldsymbol{\Omega}) I_{3}
$$

with the positive-definite expression

$$
I_{3}=\frac{8}{15 \mu_{0} \rho} \iint \frac{\nu k^{4} \mathcal{B}(k, \omega)}{\left(\omega^{2}+\nu^{2} k^{4}\right)^{2}} d \boldsymbol{k} d \omega .
$$

The kinetic helicity is thus positive (negative) at the northern (southern) hemisphere.

\section{Summary}

In the table our results are summarized for both the discussed turbulence models. In all cases the sign of the (antisymmetric) scalars are given for the northern hemisphere:

\begin{tabular}{|l||c|c|c|c|}
\hline & kinetic-driven & magnetic-driven & total & observ. \\
\hline \hline $\mathcal{H}_{\text {kin }}$ & negative & positive & $\approx 0$ & \\
\hline $\mathcal{C}$ & negative & negative & negative & $\lesssim 0$ \\
\hline $\mathcal{H}_{\text {curr }}$ & negative & negative & negative & negative \\
\hline$\alpha$-effect & positive & positive & positive & \\
\hline
\end{tabular}

If the real turbulence model can be considered as a mixture of both the approximations then we expect i) a rather small kinetic helicity $\mathcal{H}_{\text {kin }} \simeq 0$, ii) a negative current helicity $\mathcal{H}_{\text {curr }}<0$ and iii) a positive $\alpha$-effect. Only little hope remains 


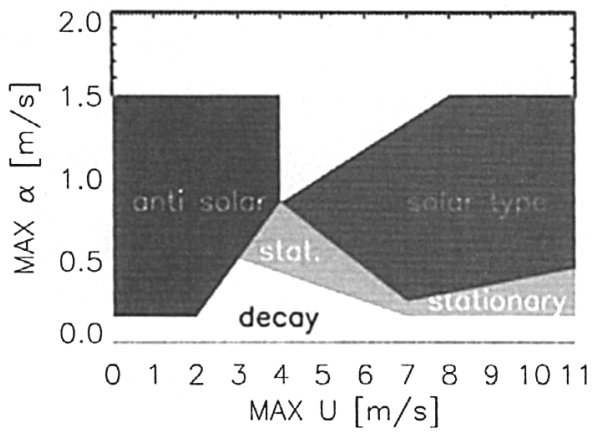

Figure 1. The dynamo regime for various amplitudes of the bottom drift (towards the equator). The activity belts are moving to the equator ('solar-type') if the drift velocity exceeds $4 \mathrm{~m} / \mathrm{s}$

to produce the negative $\alpha$-effect which is required to reproduce the observed butterfly diagram in an $\alpha \Omega$-dynamo adopting the rotation law of the solar convection zone revealed by the helioseismology. So our considerations favour the idea of the advection-dominated dynamo model incorporating meridional circulation which flows towards the equator at the bottom of the convection zone and produces the observed butterfly diagram (Choudhuri, Schüssler, \& Dikpati 1995; Dikpati \& Charbonneau 1999; Küker, Rüdiger, \& Schultz 2000). It works with a rather small magnetic diffusivity of order of $10^{11} \mathrm{~cm}^{2} / \mathrm{s}$, but the eddy viscosity must be much larger in order to understand the rotation of the solar convection zone which is not close to the Taylor-Proudman state ('low magnetic Prandtl number').

\section{References}

Abramenko, V.I., Wang, T., \& Yurchishin, V.B. 1996, Solar Phys., 168, 75

Bao, S., \& Zhang, H. 1998, ApJ, 496, L43

Brandenburg, A., \& Schmitt, D. 1998, A\&A, 338, L55

Brandt, P.N., Scharmer, G.B., Ferguson, S., \& et al. 1988, Nature, 335, 238

Choudhuri, A.R., Schüssler, M., \& Dikpati, M. 1995, A\&A, 303, L29

Dikpati, M., \& Charbonneau, P. 1999, ApJ, 518, 508

Duvall, T.L., Jr., \& Gizon, L. 2000, Solar Phys., 192, 277

Hale, G.E. 1927, Nature, 119, 708

Küker, M., Rüdiger, G., \& Schultz, M. 2000, A\&A (subm.)

Pevtsov, A.A., Canfield, R.C., \& Metcalf, T.R. 1995, ApJ, 440, L109

Rüdiger, G., Pipin, V.V., \& Belvedere, G. 2000, Solar Phys. (subm.)

Seehafer, N. 1990, Solar Phys., 125, 219

Wang, Y., Noyes, R.W., Tarbell, Th.D., \& Title, A.M. 1995, ApJ, 447, 419 